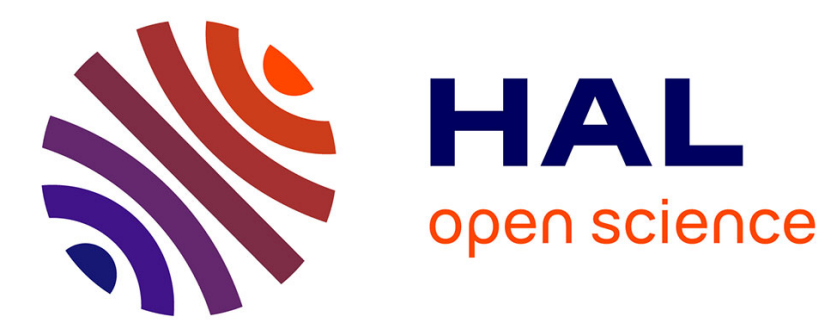

\title{
Conformation of a polymer chain in certain mixed solvents
}

P.-G. de Gennes

\section{To cite this version:}

P.-G. de Gennes. Conformation of a polymer chain in certain mixed solvents. Journal de Physique Lettres, 1976, 37 (4), pp.59-61. 10.1051/jphyslet:0197600370405900 . jpa-00231237

\section{HAL Id: jpa-00231237 https://hal.science/jpa-00231237}

Submitted on 1 Jan 1976

HAL is a multi-disciplinary open access archive for the deposit and dissemination of scientific research documents, whether they are published or not. The documents may come from teaching and research institutions in France or abroad, or from public or private research centers.
L'archive ouverte pluridisciplinaire HAL, est destinée au dépôt et à la diffusion de documents scientifiques de niveau recherche, publiés ou non, émanant des établissements d'enseignement et de recherche français ou étrangers, des laboratoires publics ou privés. 


\title{
CONFORMATION OF A POLYMER CHAIN IN CERTAIN MIXED SOLVENTS
}

\author{
P. G. DE GENNES \\ Collège de France, 75231 Paris Cedex 05, France
}

(Reçu le 23 janvier 1976, accepté le 13 février 1976)

\begin{abstract}
Résumé. - Dans un mélange $A B$ proche du point critique de démixtion, deux monomères $C_{1} C_{2}$ d'une chaîne solutée doivent avoir une interaction indirecte attractive et à portée longue (égale à la longueur de corrélation $\xi$ du système $A B$ ). Près du point critique l'interaction décroît pratiquement comme $1 / r \mathrm{e}^{-r / \xi}$. La compétition entre cette attraction et les répulsions usuelles à courte portée donne une taille anormale à la chaîne : ceci pourrait être testé par des mesures de viscosité.
\end{abstract}

\begin{abstract}
In a solvent mixture $\mathrm{AB}$ close to a critical (demixing) point $T_{\mathrm{c}}$, there should exist an indirect interaction between two solute monomers $\left(C_{1} C_{2}\right)$, which is attractive and of long range (the range being the correlation length $\xi$ of the $\mathrm{AB}$ system). Close to the critical point, the interaction is of the Coulomb form (but reversed in sign). The competition between this attraction and conventional short range repulsions leads to an anomalous size for the solute chain near $T_{\mathrm{c}}$; this could be probed through viscometric measurements.
\end{abstract}

1. Introduction. - The behaviour of polystyrene chains in a mixture of one good and one bad solvent has been investigated experimentally and theoretically by Dondos and Benoit [1] : they showed that the solvent composition near the chain is modified.

The present letter represents a slight extension of their work, on the theoretical side, to cover one special situation. Namely, we consider the extreme case of two solvents A, B which are nearly incompatible, with a critical point as shown on figure 1 . In this case the weak perturbations induced by a solute chain can have dramatic effects.

The properties of critical points in binary fluids have been investigated in considerable detail $[2,3]$ : the main feature is the existence of large fluctuations of the relative concentration $c=A / A+B$; they take place over a characteristic distance $\xi(c, T)$ which becomes very large near the critical point. (We have plotted on figure 1 a few lines of constant $\xi$ - isorange lines - in a $(c, T)$ diagram). With moderate temperature stabilizations it is quite feasible to make $\xi$ comparable or larger than a coil size - hence the interest. Note the following features of the isorange curves.

- The distance from the critical point $O P=\Delta T$ (measured along the temperature axis) is related to $\xi$ by $\xi=$ const $\Delta T^{-v}$ where $v$ is a critical exponent defined in [2] $\left(v \sim \frac{2}{3}\right)$.

- The distance $O Q=\Delta C$ (measured along the concentration axis) is given by $\xi=$ const $\Delta C^{-v / \beta}$ where $\beta$ is another critical exponent [2] $\left(\beta \sim \frac{1}{3}\right)$.

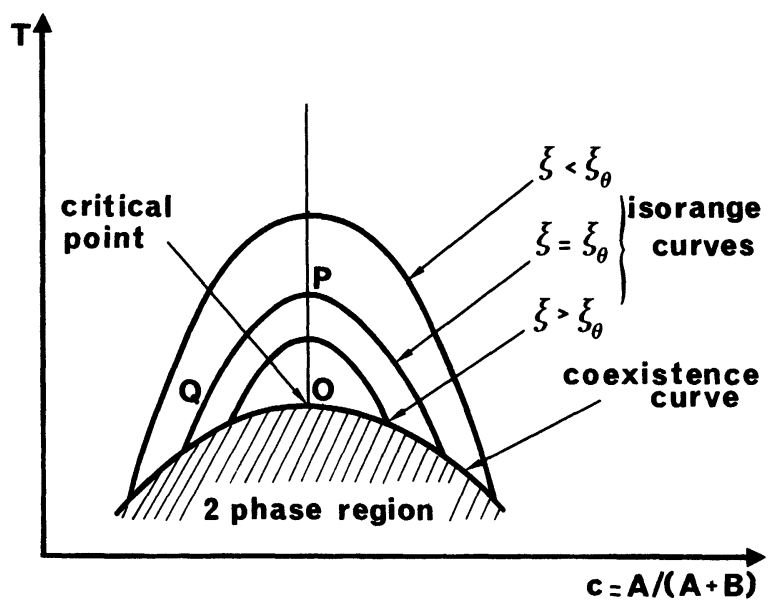

FIG. 1.

2. Structure of the long range interaction. - Let us consider first one single monomer (i) located at $\mathbf{r}_{i}$ in the mixed solvent $(A+B)$. The average concentration of $A$ is $c$ but it has local fluctuations $\delta c(r)$. The chemical potential of one monomer in the medium is of the form

$$
\varepsilon=\varepsilon_{0}+\varepsilon_{1} \delta c\left(\mathbf{r}_{i}\right)
$$

where $\varepsilon_{1}$ may be of arbitrary sign. If $\varepsilon_{1}$ is positive (negative) the local fraction of $A$ around $\mathbf{r}_{i}$ will be decreased (increased). This is described by a response function

$$
\delta c(\mathbf{r})=-\chi\left(\mathbf{r}-\mathbf{r}_{i}\right) \varepsilon_{1} .
$$


The integral over space of $\delta c$ is essentially the parameter of preferential solvation $\lambda^{\prime}$ of reference [1]

$$
\lambda^{\prime}=\varepsilon_{1} \int \chi\left(\mathbf{r}-\mathbf{r}_{1}\right) \mathrm{d} \mathbf{r}=\varepsilon_{1} \bar{\chi} .
$$

To a good approximation $\chi(\mathbf{r})$ has a simple OrnsteinZernike form

$$
\chi(\mathbf{r})=\frac{\bar{\chi}}{4 \pi \xi^{2}} \frac{1}{r} \mathrm{e}^{-r / \xi} .
$$

Eq. (4) is rigorous for $r>\xi$. For $r<\xi$ it neglects the $\eta$ correction of Fisher [2] : but this correction has never been large enough to be detected in threedimensional phase transitions. Thus eq. (4) is acceptable. To the same approximation, the coefficient $\bar{\chi} / \xi^{2}$ is finite and essentially independent of $T$ and $c$ near the critical point.

Returning now to the effects around monomer (i), let us now add a second monomer $(j)$ at some distance : it will feel a concentration shift $\delta c\left(\mathbf{r}_{j}\right)$ and a change of energy

$$
\left.\begin{array}{rl}
W_{1}\left(\mathbf{r}_{i j}\right) & =\varepsilon_{1} \delta c\left(\mathbf{r}_{j}\right)=-\varepsilon_{1}^{2} \chi\left(\mathbf{r}_{i}-\mathbf{r}_{j}\right)=\frac{-e^{2}}{r_{i j}} \mathrm{e}^{-r_{i j} / \xi} \\
e^{2} & =\frac{\varepsilon_{1}^{2} \pi}{4 \pi \xi^{2}}
\end{array}\right\}
$$

The interaction $W_{1}$ is always attractive; except for the sign, it is similar to a screened Coulomb force (hence the notation used in the last form of [5]).

3. Two good solvents. - In practice, we will have, in addition to $W_{1}$, the usual short range forces $W_{2}$ between monomers, which can be described by a conventional excluded volume parameter $v$ (proportional to the $\frac{1}{2}-\chi$ ) of Flory [3]. We shall assume that $v$ is positive and fixed in the $(c, T)$ region of interest; this is acceptable if both $A$ and $B$ are good solvents for the chain. The total monomer-monomer interaction is then

$$
W\left(\mathbf{r}_{i j}\right)=W_{1}+W_{2}=\frac{-e^{2}}{r_{i j}} \mathrm{e}^{-r_{i j} / \xi}+T v \delta\left(\mathbf{r}_{i j}\right) .
$$

It is of interest to consider first the space integral of this interaction

$$
v_{\text {eff }}=\frac{1}{T} \int W\left(\mathbf{r}_{i j}\right) \mathrm{d} \mathbf{r}_{i j}=v-4 \pi e^{2} \xi^{2} .
$$

There is one special isorange curve for which this integral vanishes, we call it the theta curve ; the corresponding value of $\xi$ is

$$
\xi_{\theta}=\left(\frac{T v}{4 \pi}\right)^{1 / 2} \frac{1}{e} .
$$

Note that $\xi_{\theta}$ is independent of the molecular mass. If both indirect and steric interactions are strong, $\xi_{0}$ will be comparable to a monomer size $a$. But if the indirect interactions are somewhat weaker than the steric effects, $\xi_{\theta}$ will be larger than $a$. We shall consider mostly this limit, where a simple continuum description is meaningful.

Below the $\theta$ curve, we expect that solute chains (even if they are very dilute) will precipitate [3]. Thus practical experimentation will be restricted to the domain above the $\theta$ curve in figure 1 , which we shall discuss now. Following the lines of references $[4,5,3]$, i.e. assuming a gaussian coil of dilation factor $\alpha=R / R_{0}$ and neglecting all correlations between monomers, one arrives at a free energy per chain $F$ of the form

$$
\frac{F}{T}=3\left(\frac{\alpha^{2}}{2}-\ln \alpha\right)+\frac{X}{\alpha^{3}}\left[1-\frac{\xi^{2}}{\xi_{\theta}^{2}} f\left(\frac{\alpha R_{0}}{\xi}\right)\right]
$$

where

$$
X=\frac{27}{2}(2 \pi)^{-3 / 2} v_{0} N^{1 / 2} a^{-3}=k_{0} v_{0} a^{-3} N^{1 / 2},
$$

$N$ being the polymerization index and $R_{0}=N^{1 / 2} a$ the unperturbed radius. The function $f$ describes the effect of the long range interactions, and interpolates smoothly between the following limits :

$\left.\begin{array}{lll}U \rightarrow 0 & f(U) \rightarrow \frac{1}{9} U^{2} & \text { (unscreened) } \\ U \rightarrow \infty & f(U) \rightarrow 1 & \text { (strong screening) }\end{array}\right\}$.

Minimizing (9) with respect to $\alpha$ one arrives at

$$
\begin{gathered}
X=\frac{\alpha^{5}-\alpha^{3}}{1-\frac{\xi^{2}}{\xi_{\theta}^{2}} g\left(\frac{\alpha R_{0}}{\xi}\right)} \\
g(u)=f(u)+\frac{1}{3} u f^{\prime}(u) .
\end{gathered}
$$

The function $g$ is similar in shape to $f(u): a$ ) For large chains $(R>\xi) g$ is equal to one and eq. (11) describes simply the replacement $v \rightarrow v_{\text {eff }}$ in the Flory equation [3]; $b)$ For smaller chains $(R \ll \xi)$

$$
X \Rightarrow \frac{\alpha^{5}-\alpha^{3}}{1-\frac{5}{27} \alpha^{2} \frac{R_{0}^{2}}{\xi_{\theta}^{2}}} .
$$

Note that $\alpha R_{0} \ll \xi$ implies $\alpha R_{0} \gg \xi_{\theta}$ since $\xi<\xi_{\theta}$ above the $\theta$ curve. Thus the correction described by the denominator is never catastrophic in this domain $\left({ }^{1}\right)$.

4. Solvents of opposite qualities. - Let us now assume that $A$ is a good solvent for the chains, while $B$

( $\left.{ }^{1}\right)$ Below the $\theta$ curve, if it was possible to observe a single chain (i.e., to avoid precipitation) eq. (12) would predict a radius $R \sim \xi_{\theta}$ independent of $N$ (for large $N$ ) and independent of the distance to the critical point ! 
is a poor solvent. This was the case in most of the experiments of reference [1]. Here we meet another effect, which was already described in [1] : we first note that the coil volume becomes enriched in solvent $A$. The concentration shift being

$$
\bar{\delta} c=\varepsilon_{1} \bar{\chi} \rho=\lambda^{\prime} \rho,
$$

where $\rho \cong N / R^{3}$ is the number density of monomers inside the coil. The excluded volume parameter $v$ increases with the quality of the solvent. This may be written as

$$
v \rightarrow v(c T)+\frac{\partial v}{\partial c}(c T) \bar{\delta} c+\cdots=v+v^{\prime} \varepsilon_{1} \chi \rho .
$$

Eqs. (13-14) assume that :

a) The density $\rho$ is slowly varying on distances of order $\xi$, i.e. that $R \gg \xi$ : in this section, for simplicity, we consider only the limit of large coils.

b) The correction $\delta c$ is not too large, i.e. that

$$
\varepsilon_{1} \bar{\chi} \rho \sim \varepsilon_{1} \bar{\chi} N R^{-3} \approx c .
$$

The effect of eq. (14) is to favor an extra swelling of the chain, and is thus opposite in sign to the effect of the indirect interaction [5]. To reach some understanding of their respective role, let us rewrite the interaction free energy (per $\left.\mathrm{cm}^{3}\right) F_{\text {int }}$ as a function of $\rho$ :

$$
\begin{aligned}
\frac{F_{\text {int }}}{T} & =\frac{1}{2} \rho^{2}\left[v+v^{\prime} \delta \bar{c}-4 \pi e^{2} \xi^{2}\right]+W \rho^{3}+\cdots \\
& =\frac{1}{2} v_{\text {eff }} \rho^{2}+\left[\frac{1}{2} v^{\prime} \varepsilon_{1} \bar{\chi}+W\right] \rho^{3}+\cdots
\end{aligned}
$$

where the last term $\left(W \rho^{3}\right)$ comes from short range interactions between three monomers $\left(W^{1 / 2}\right.$ is comparable to the monomer volume).
Thus the effect of a finite $\partial v / \partial c=v^{\prime}$ is only to renormalize the $\rho^{3}$ terms. Whenever the inequality (15) holds this renormalization is weak. Finally, for positive $v_{\text {eff }}$, or even for $v_{\text {eff }} \rightarrow 0$, we know that the cubic terms are not essential for a calculation of the coil size [6]. Thus the conclusions of section 3 are unaltered whenever (15) is satisfied. Dimensionally, for conditions near the $\theta$ curve, the inequality (15) may be transformed into

$$
R_{0}>\xi_{\mathrm{s}}^{2} / a
$$

where $a$ is a monomer size.

For smaller chains $\left(R_{0}<\xi_{\mathrm{s}}^{2} / a\right)$ the density $\rho$ is larger and the linear response eqs. (13-14) fail : $\bar{\delta} c$ will be limited by saturation effects. We conclude that for most practical situations near a critical point the dominant effect should be due to the attractive indirect interactions. This is very different from the situation studied in reference [1].

5. Concluding remarks. - It may be feasible to study the behaviour described by eqs. (11-12) with dilute chains in suitable solvents, light scattering methods are not adequate (since critical scattering will dominate) but viscometric studies of $R$ are acceptable : the anomalies in the solvent viscosity near a demixing critical point are weak [7].

Finally, another interesting class of problems could be found if the chain, instead of being neutral as assumed above, was in fact a poly-electrolyte : then the indirect interaction would counteract the real Coulomb repulsion, and lead to an apparent charge which would be reduced.

Acknowledgments. - It is a pleasure to thank Prof. P. A. Pincus for various discussions.

\section{References}

[1] Dondos, A., Benort, H., J. Polym. Sci. B 7 (1969) 335 ; Macromol. Chem. 133 (1970) 119.

[2] For a general review on critical points see : Critical phenomena Proceedings of the Varenna Summer School 1970 No 51 edited by M. S. Green (Academic Press, N. Y.) 1971 ; in particular courses by M. Fisher, M. Vicentini-Missoni and $\mathrm{K}$. Kawasaki.

[3] Flory, P., Principles of polymer chemistry, Cornell Un. Press Ithaca (N. Y.).
[4] Hermans, J. J., Overbeek, J. T., Rec. Trav. Chim. 67 (1948) 761. [5] Kuhn, W., Kunzle, D., Katchalsky, A., Helv. Chim. Acta 31 (1948) 1994.

[6] Stephen, M., Phys. Lett. 53A (1975) 363.

[7] Stein, A., Allegra, J., Allen, G., J. Chem. Phys. 55 (1971) 4265.

Chang, R., Keyes, P., Sengers, J., Alley, C., Phys. Rev. Lett. 27 (1971) 1706. 